\title{
*1PROSTATE CANCER SCREENING IN GHANA - A CLINICAL BENEFIT?
}

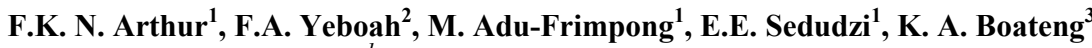 \\ ${ }^{1}$ Department of Biochemistry, \\ Kwame Nkrumah University of Science and Technology, Kumasi \\ ${ }^{2}$ Department of Molecular Medicine, School of Medical Sciences, \\ Kwame Nkrumah University of Science and Technology, Kumasi \\ ${ }^{3}$ Department of Pathology, School of Medical Sciences, \\ Kwame Nkrumah University of Science and Technology, Kumasi
}

\begin{abstract}
In Ghana and most African countries, prostate cancer is the most common cancer in males after hepatocellular carcinoma. Whereas in the advanced countries, screening for prostate specific antigen (PSA) has led to early detection and management of the disease, screening has been very low in Ghana, thus leading to low detection rate, poor management and increased mortality. The aim of our study therefore was to provide the basis for the advocacy and promotion of prostate cancer screening in Ghana so as to improve early detection, management and prognosis. To do this, we estimated serum PSA levels of 196 men randomly reporting to the Out-Patient Department (OPD) of the Komfo Anokye Teaching Hospital for screening. We employed the enzyme immunoassay (EIA) method as our non-invasive screening procedure. We also conducted a survey on the prevalence of the disease as reported at five major hospitals in the country. It was observed that $83.6 \%$ of the subjects had their PSA levels above the upper limit of the reference range $(4 \mathrm{ng} / \mathrm{ml})$, with their ages ranging between 56 to 85 years. Coincidentally, our survey also revealed that $96.5 \%$ of those who had the disease within the period of study were above 50 years. The significance and relevance of these findings to PSA screening in the country and its accuracy in diagnosing prostate cancer cannot be overemphasized.
\end{abstract}

Keywords: Prostate cancer, screening, diagnosis, prostate specific antigen (PSA)

\section{INTRODUCTION}

The prostate is a small gland situated at the base of the penis, just below the bladder and in front of the rectum. The size of the gland at birth is like a small pea and remains so until puberty when there is a spurt of growth as it starts producing hormones. It remains so till about middle age, when it begins gradually to increase in size, probably due to hormonal changes that occur with age. Some men may develop benign prostatic hyperplasia (BPH); even though not cancerous, the organ increases in size and puts pressure on the urethra, making urination difficult. Others may develop prostatic intraepithelial neoplasia (PIN), which predisposes individuals to prostate cancer. Prostate cancer, in its earliest stage may produce no sign or symptoms; as the tumor grows, certain signs or symptoms may be noticed which include painful or burning urination, frequent urination, painful ejaculation and blood in urine. Other symptoms include swelling in the groin area, loss of appetite and weight loss, and impotence. These symptoms can also be caused by $\mathrm{BPH}$ and prostatitis. In most cases, by the time these symptoms appear, the cancer has moved beyond the prostate gland, making treatment much more difficult.

Prostate cancer is the most common cancer in males after hepatocellular carcinoma in Africa (Parkin, 2003), but in the United States and Northwestern Europe, it is the most common male cancer and the second most common cause of cancer deaths (Jemal et al., 2002). In contrast, its incidence is much lower in Asia, South America and Africa. In African countries where registers exist such as Nigeria, Uganda, South Africa and Zimbabwe, it has been observed that the incidence of prostate cancer is increasing between the ages of 40 to 70 years (Wabinga, 2003).

In Ghana, no cancer register has ever been published because there is no available statistics on the disease.

${ }^{1}$ *This article is being republished because some tables were left out in the Vol. 25 No. 2, 2005 
Preliminary hospital records indicate that the incidence of prostate cancer has been observed to be increasing, although there are no statistics to prove it. In order to advocate and promote PSA screening among the older population, we decided to perform PSA screening among one hundred and ninety-six (196) consented men, randomly selected from various parts of Kumasi. We also conducted a survey on the prevalence in various parts of the country to determine the age-group that is mostly at risk.

At present, there are no clear strategies to prevent prostate cancer through life-style modification or preventive intervention and therefore, an early non-invasive screening will be highly beneficial as a precautionary measure against the onset of the disease.

\section{MATERIALS AND METHODS}

PSA Screening

The enzyme immunoassay (EIA) was adopted for the measurement of PSA. In this method, the serum test sample was allowed to react first with the immobilized rabbit antibody at room temperature for 60 minutes. The wells were washed to remove any unbound antigen. The monoclonal anti-PSA horseradish peroxidase conjugate was then reacted with the immobilized antigen for 60 minutes at room temperature resulting in the PSA molecules being sandwiched between the solid phase and the enzyme-linked antibodies. The wells were washed with water to remove unbound-labelled antibodies. A solution of Tris-buffer was added and incubated at room temperature for 20 minutes resulting in the development of a blue colour which was stopped with the addition of $1 \mathrm{~N} \mathrm{HCl}$. The resultant yellow chromophore was read at $450 \mathrm{~nm}$.

\section{Prostate Cancer Survey}

A questionnaire was sent out to the records department of five prominent health institutions in Ghana after obtaining permission from the health authorities. These institutions were Korle-Bu Teaching Hospital, Accra, Komfo Anokye Teaching Hospital, Kumasi, Effia Nkwanta Hospital, Takoradi, Cape Coast Regional Hospital and Sunyani Regional Hospital. The questionnaire covered all prostate cancer cases, both out-patients and in-patients between the period from 1998 to 2003. The following information was sought:

(a) Age at first diagnosis, and date of diagnosis.

(b) Place of residence and occupation.

(c) Treatment regime and prognosis.

The questionnaire were filled in by the biostatisticians at the records department at the hospitals and the data were collated and results analyzed by Excel software package.

Table 1 Mean PSA Levels against age-groups

\begin{tabular}{ccccccc}
\hline $\begin{array}{c}\text { AGE GROUPS } \\
\text { (YRS) }\end{array}$ & $<$ 4ng/ml & $\mathbf{4 - 1 0 n g / m l}$ & $\mathbf{1 1 - 4 0 n g} / \mathbf{m l}$ & $\begin{array}{c}\text { Mean PSA } \\
\text { Values }\end{array}$ & TOTAL & \% TOTAL \\
\hline $41-45$ & 5 & 0 & 0 & 0.68 & 5 & 3.2 \\
$46-50$ & 7 & 1 & 1 & 9.83 & 10 & 6.3 \\
$51-55$ & 3 & 0 & 0 & 4.32 & 5 & 3.2 \\
$56-60$ & 12 & 3 & 3 & 16.44 & 23 & 14.6 \\
$61-65$ & 5 & 6 & 6 & 28.58 & 20 & 12.7 \\
$66-70$ & 8 & 13 & 13 & 37.83 & 32 & 20.3 \\
$71-75$ & 8 & 9 & 10 & 31.13 & 26 & 16.5 \\
$76-80$ & 4 & 7 & 7 & 27.77 & 21 & 13.3 \\
$81-85$ & 2 & 5 & 5 & 33.96 & 8 & 5.1 \\
$86-90$ & 1 & 1 & 1 & 47.78 & 4 & 2.5 \\
$91-95$ & 1 & 3 & 3 & 74.25 & 4 & 2.5 \\
TOTAL No OF & 56 & 48 & 49 & 47 & 158 & \\
SUBJECTS & & & & & & \\
\hline
\end{tabular}


Figure 1: PSA Levels against Age Groups

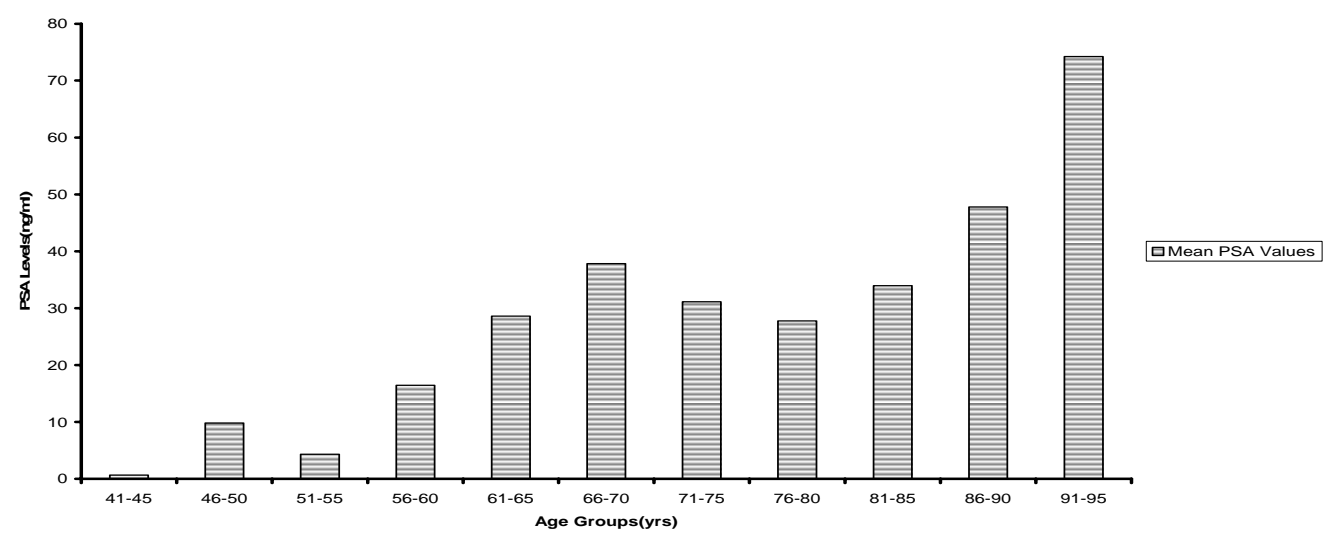

\section{RESULTS AND DISCUSSION}

The overall results from our screening studies are shown below in Figures 1-4. PSA measurements were performed on one hundred and ninety-six (196) subjects, aged between 40 to 95 years, by random selection. The values ranged from 0 to $110 \mathrm{ng} / \mathrm{ml}$. Generally, serum PSA was observed to increase with age as shown in Figure 1. Table 1 and Figures 2-4 show the PSA levels (4-10) ng/ml, (11-40) ng/ml, and (>40) ng/ml, against age groups of subjects. It was observed that 56 out of 196 patients or approximately $28.5 \%$ had their PSA levels below $4 \mathrm{ng} / \mathrm{ml}$, indicating the possible absence of prostatic disease in this group of subjects. It was also found that about $24.4 \%$ of those screened had their PSA levels in the range of $4-10$ $\mathrm{ng} / \mathrm{ml}$, and of this number, only one or $0.5 \%$ was below 50 years, while 9 or $4.5 \%$ were above 80 years. These results do not necessarily rule out the complete absence of prostate carcinoma, since they may have a latent stage of the disease or a localized tumor or even BPH which often leads to increased amount of PSA in serum. The data in Table 1 also shows that $25.0 \%$ of the subjects had their PSA levels in the range of 11$40 \mathrm{ng} / \mathrm{ml}$ and only one or $0.5 \%$ was below 50 years while nine or $4.5 \%$ were above 80 years. A relatively high incidence of the disease may occur in this PSA range and may show symptoms of the disease. $23.4 \%$ of studied subjects had their PSA levels $>40 \mathrm{ng} / \mathrm{ml}$ and of these, only one or $0.5 \%$ was below 50 years, while 8 or $4.0 \%$ were above 80 years. Subjects in this PSA range could have the highest incidence of the disease which may have metastasized to the fatal stage. It is also evident from Table 1 that majority or 164 of the subjects $(80.4 \%)$ who had their PSA levels higher than the normal $(4 \mathrm{ng} / \mathrm{ml})$ value were between ages 56 to 85 years. It should however, be mentioned here that PSA as a non-invasive diagnostic screening procedure is not $100 \%$ sensitive.

Figure 2: Patients with PSA level 4 10ng/ml against Age Groups

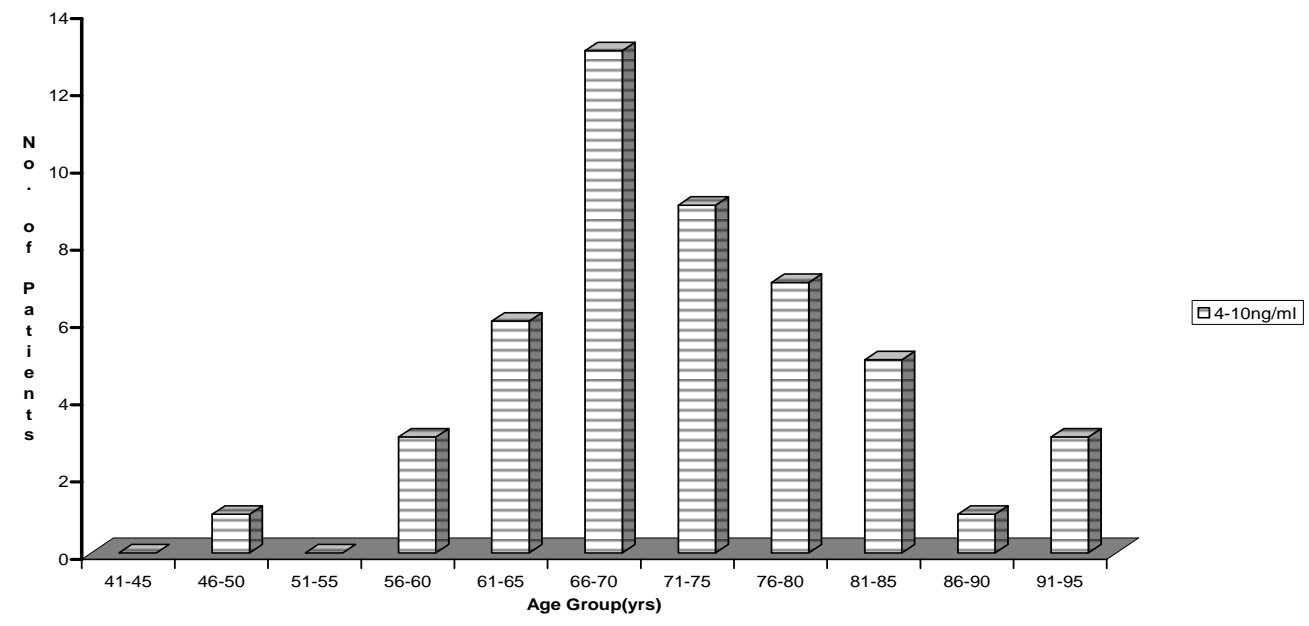


Figure 3: Patients with PSA level (11-40 ng/ml) against age Groups

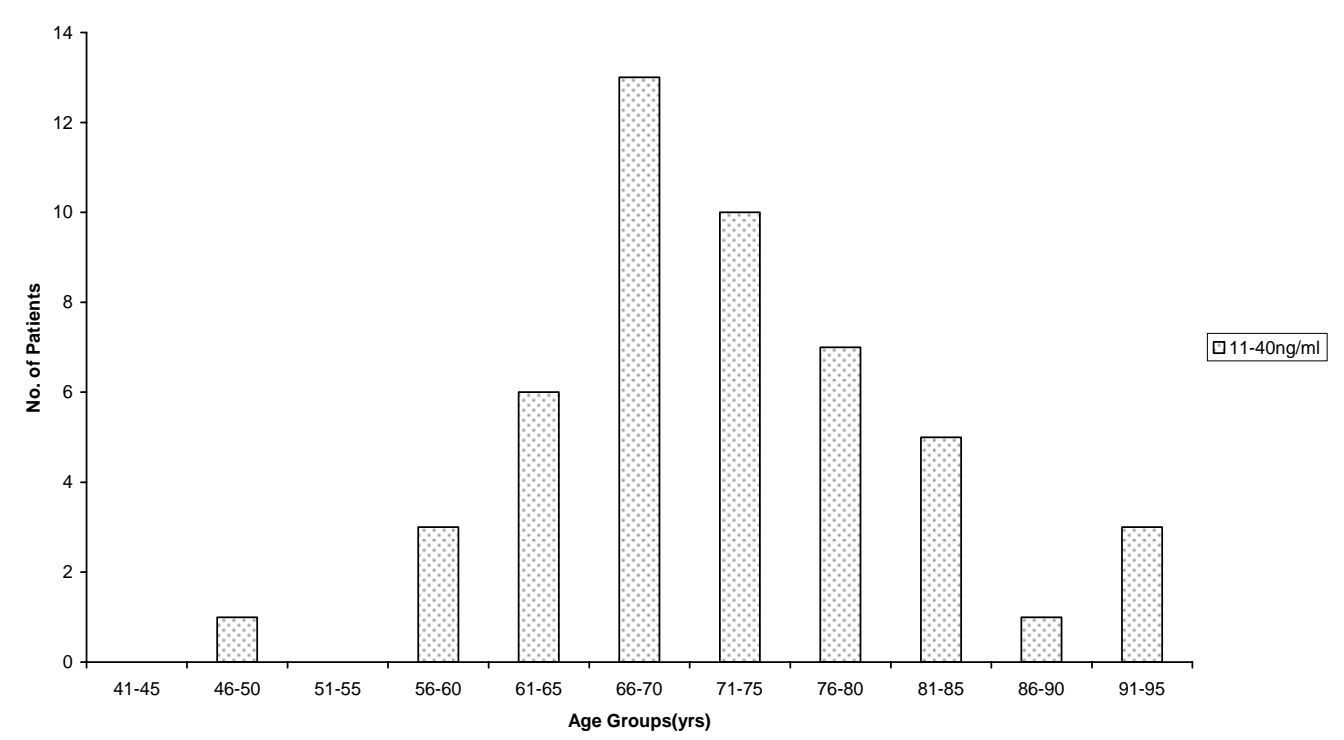

Figure 5 shows the number of prostate cancer cases against the age group of patients as obtained from the records department of five major hospitals in the country. The period under study was 1998 to 2003 . The total number of cases recorded was 512 . Over $96 \%$ of those subjects were above 50years and only 18 cases or $3.5 \%$ occurred within ages $9-49$ years. Incidentally, Table 1 also shows that majority of the patients with abnormally high PSA level occurred within the 51-80 years age group.

Prostate specific antigen is a glycoprotein produced by the prostatic epithelial cells and has a molecular weight of about $33 \mathrm{kd}$. It can be detected in the blood of all adult men; its level increases in men with prostate cancer and other disorders of the prostate including prostatitis and benign prostatic hypertrophy (BPH). Normal total PSA levels are between zero and four nanogram per milliliter $(0-4 \mathrm{ng} / \mathrm{ml})$. It has been reported that between 1989 and 1992 when PSA testing first became widely available in the US, the reported incidence of the disease rose to nearly $70 \%$ (PCC, 2001). This clearly emphasizes the importance of PSA testing to the detection of the cancer. In the Western countries, men diagnosed with prostate cancer in recent years have more aggressive and better treatment options than two decades ago. The obvious question to ask is "Is their chance of dying of the disease dropping as a result?" In a preliminary report following an Austrian study, an aggressive program of PSA screening and cancer treatment has been credited with a $42 \%$ reduction in population-based mortality in 1998 against a base period of 1986 to 1990 . About 65,000 men aged between the ages of 45 to 75 were involved (PCC, 2001). In a Canadian study, $69 \%$ reduction in mortality in men aged between 45 and 80 who participated in screening was achieved, as opposed to those who did not. The investigators used $3 \mathrm{ng} / \mathrm{ml}$ as the upper limit of normal for PSA (ONI, 1998). Also, in a report released by the United States of America Preventive Services (USAPS) Task Force on prostate cancer, it was stated that there had been a reduction of mortality which could be due to the screening for serum PSA, although the effectiveness of screening in reducing prostate cancer mortality has not been established (USPS Task Force, 2002).

This underscores the relevance of PSA testing to early detection of the disease and there is no question that the testing has revolutionized the detection of early-stage prostate cancer, which in most cases is amenable to successful treatment. In Ghana, prostate cancer is mostly presented at the hospital when the disease is advanced and has metastasized to other vital organs, a stage which then becomes difficult, if not impossible to treat and may be fatal. This is mainly due to ignorance of the disease, and the likelihood of the individual wanting to seek alternative forms of treatment, which invariably, does not improve the condition. In most situations, digital rectal examination (DRE) and PSA testing by the doctor are discretionary, and irregular and rarely, a screening exercise. It is believed that introduction of PSA screening will raise awareness and thus help improve early detection and management of the disease. At present, it costs about $₫ 50,000.00$ 
(fifty thousand cedis) per test and it could be considerably lower if it becomes widely available.

CONCLUSION

Our study has clearly indicated that about $86.6 \%$ of the subjects who had their PSA levels higher than normal were between ages 56-85 years. Coincidentally, it was in this age group that we observed the highest prevalence $(96.5 \%)$ of prostate cancer in the survey that was conducted.

We therefore advocate that voluntary annual PSA screening should be recommended for males aged 50 years and above, including those in the high risk bracket. There is also the need for increased awareness of the factors that predispose people to the disease. The significance and relevance of these findings to PSA screening in the country and its accuracy in diagnosing prostate cancer cannot be overemphasized.

\section{REFERENCES}

Jemal, A., Thomas, A., Murray, T., Thun, M. (2002). Cancer Statistics. California Cancer Journal for Clinicians 52: 23-47.

Oncology News International (ONI) Report. Vol. 7, No 7 (July 1998).

Parkin, D. M. (2003). Epidemiology of cancer in Africa. (Abstract). Proceedings of the $4^{\text {th }}$ International Conference on Cancer in Africa. Accra, 6-10 October.

Primary Care and Cancer (PCC) Report. Vol. 21, No 6 (June 2001).

United States of America Preventive Services (USAPS) Task Force (2002). Screening for prostate cancer: Recommendation and Rationale. Ann. Intern. Med. 137: 915-916.

Wabinga, H. (2003). Cancer registry development in Africa. (Abstract). Proceedings of the $4^{\text {th }}$ International Conference on Cancer in Africa. Accra, October 6-10. 